\title{
Reductions of copper ion-mediated low-density lipoprotein (LDL) oxidations of trypsin inhibitors, the sweet potato root major proteins, and LDL binding capacities
}

\author{
Yeh-Lin Lu ${ }^{1 \dagger}$, Chia-Jung Lee ${ }^{2 \dagger}$, Shyr-Yi Lin ${ }^{3,4^{*}}$ and Wen-Chi Hou ${ }^{5^{*}}$ (])
}

\begin{abstract}
Background: The root major proteins of sweet potato trypsin inhibitors (SPTIs) or named sporamin, estimated for 60 to $80 \%$ water-soluble proteins, exhibited many biological activities. The human low-density lipoprotein $(\mathrm{LDL})$ showed to form in vivo complex with endogenous oxidized alpha-1-antitrypsin. Little is known concerning the interactions between SPTIs and LDL in vitro.

Results: The thiobarbituric-acid-reactive-substance (TBARS) assays were used to monitor $0.1 \mathrm{mM} \mathrm{Cu}^{2+}$-mediated low-density lipoprotein (LDL) oxidations during 24-h reactions with or without SPTIs additions. The protein stains in native PAGE gels were used to identify the bindings between native or reduced forms of SPTIs or soybean TIs and LDL, or oxidized LDL (oxLDL). It was found that the SPTIs additions showed to reduce LDL oxidations in the first 6-h and then gradually decreased the capacities of anti-LDL oxidations. The protein stains in native PAGE gels showed more intense LDL bands in the presence of SPTIs, and 0.5 -h and 1-h reached the highest one. The SPTIs also bound to the $\mathrm{OxLDL}$, and low $\mathrm{pH}$ condition ( $\mathrm{pH}$ 2.0) might break the interactions revealed by HPLC. The LDL or oxLDL adsorbed onto self-prepared SPTIs-affinity column and some components were eluted by $0.2 \mathrm{M} \mathrm{KCl}(\mathrm{pH}$ 2.0). The native or reduced SPTIs or soybean TIs showed different binding capacities toward LDL and oxLDL in vitro.
\end{abstract}

Conclusion: The SPTIs might be useful in developing functional foods as antioxidant and nutrient supplements, and the physiological roles of SPTIs-LDL and SPTIS-OxLDL complex in vivo will investigate further using animal models.

Keywords: Low-density lipoprotein (LDL), Thiobarbituric-acid-reactive-substance (TBARS), Sweet potato trypsin inhibitors (SPTIS)

\section{Background}

Sweet potato (SP) (Ipomoea batatas [L.] Lam) is ranked the 3rd productions after potato and cassava among the five major tuberous crops (potato, cassava, SP, yam, and

\footnotetext{
*Correspondence: sylin@tmu.edu.tw; wchou@tmu.edu.tw

${ }^{\dagger}$ Yeh-Lin Lu and Chia-Jung Lee contributed equally to this study

${ }^{4}$ Department of General Medicine, School of Medicine, College

of Medicine, Taipei Medical University, Taipei, Taiwan

${ }^{5}$ Graduate Institute of Pharmacognosy, Taipei Medical University, No. 250,

Wu-Hsing Street, Taipei 110, Taiwan

Full list of author information is available at the end of the article
}

taro) in the world, which account for about $99 \%$ total productions of tuberous crops worldwide (FAO 1999; Shewry 2003). The nutritional values of carbohydrates in tuberous crops are mainly for dietary energy supplies; and the approximate protein contents are $3-6 \%, 1-10 \%$, and $1-2 \%$ of dry weight, respectively, for potato, SP, and cassava (Shewry 2003), or varies among $1-2 \mathrm{~g}$ per 100 -g edible portion (FAO 1990). SP roots also contain higher dietary fibers, beta-carotene, and vitamins (especial for thiamine, ascorbic acid and folic acid) among major tuberous crops, which are used as staple foods or foods, animal feeds, and industrial starch processing (Mohanraj 
and Sivasankar 2014; Heuzé et al. 2015). Except roots, SP leaves contains phenolic compounds, such as lutein, quercetin, caffeoylquinic acid derivatives, and chlorogenic acid, which provide protection against aging, cancer, allergies, diabetes, hypertension, and cardiovascular diseases (Mohanraj and Sivasankar 2014).

Sohonie and Bhandarker (1954) described the trypsin inhibitory activities in SP roots, which was the first report in non-leguminous plants. Lin and Chen (1980) reported that the trypsin inhibitory activities of water extracts from 53 varieties of SP roots in Taiwan exhibited heatresistant and heat-labile properties, which the trypsin inhibitory activities might be as useful taxonomic traits in SP varieties (Lin 1988). Maeshima et al. (1985) purified and named sporamin as the major storage proteins in SP roots. Later, the overexpressed sporamin in E. coli showed trypsin inhibitory activity (Yeh et al. 1997; Senthilkumar and Yeh 2012). The purified SPTIs have been reported to exhibit in vitro biological activities, such as dehydroascorbate eductase and momodehydroascorbate reductase activities (Hou and Lin 1997a), antioxidant activities (Hou et al. 2001, 2005), glutathione peroxidaselike activities (Hou et al. 2004), angiotensin converting enzyme inhibitory activities (Huang et al. 2006), thioltransferase-like and glutathione S-transferase-like activities (Huang et al. 2009). Huang et al. (2008) reported that $\mathrm{BALB} / \mathrm{c}$ mice fed normal diet concurrent with SPTIs $(100 \mathrm{mg} / \mathrm{kg})$ by gavaging once every 2 days for 35 days. They found that the SPTIs interventions showed significantly to increase plasma antioxidant activities by ABTS method and reduce hepatic malondialdehyde (MDA) and plasma triglyceride levels compared to the control $(P<0.05$ or 0.01$)$, however, no significant change of plasma low-density-lipoproteins (LDL)-cholesterol (C) level was reported. Ishiguro et al. (2016) used three heatstable proteases at $\mathrm{pH} 8.5,65^{\circ} \mathrm{C}$ for $16 \mathrm{~h}$ to treat SP proteins in order to get SP peptides (SPP). The BALB/c mice fed high-fat diet containing $0.5 \%$ or $5 \%$ SPP (W/W of high-fat diet) for 28 days significantly showed to reduce body weight, epididymal fats and mesenteric fats, plasma triglyceride, and LDL-C compared to those in the control fed high-fat diet only.

The oxidized LDL (oxLDL) is an important factor in atherosclerotic pathogenesis (Kawamura et al. 1994). Therefore, $\mathrm{Cu}^{2+}$-mediated LDL oxidation was a general method in vitro to investigate effects of natural compounds or extracts on delaying LDL oxidations, which the associated mechanisms might involve $\mathrm{Cu}^{2+}$-mediated destructions of $\operatorname{Trp}$ residues in apoB portion of LDL to generate Trp radicals, which participated in advance to unsaturated lipid oxidations (Gießauf et al. (1995); Schnitzer et al. 1997; Knott et al. 2002; Nakano et al. 2004). Wu et al. (2009) reported that the tested phenolic compounds, such as luteolin, naringenin, kaempferol, quercetin, showed to bind LDL and enhance in vitro protections against $\mathrm{Cu}^{2+}$-mediated LDL oxidations detected by thiobarbituric-acid-reactive-substance (TBARS) assays. Wu et al. (2009) reported that the tested phenolic compounds, such as luteolin, naringenin, kaempferol, quercetin, showed to bind LDL and enhance in vitro protections against $\mathrm{Cu}^{2+}$-mediated LDL oxidations detected by thiobarbituric-acid-reactive-substance (TBARS) assays. Mashiba et al. (2001) reported that human oxidized (inactivated) plasma $\alpha_{1}$-antitrypsin (oxidized AT) could form complex with LDL and co-eluted in the gel permeation chromatography, and this complex might play anti-atherosclerotic effects in vivo (Kotani et al. 2010). Therefore, in this study, the TBARS assays were used to monitor $0.1 \mathrm{mM} \mathrm{Cu}^{2+}$-mediated LDL oxidations during 24-h reactions with or without SPTIs additions. The protein stains in native PAGE gels were used to identify the bindings between native or reduced forms of SPTIs and LDL, oxLDL, and commercial soybean TIs were used for comparisons.

\section{Methods \\ Materials}

Copper (II) sulfate, 2-thiobarbituric acid, and trypsin (TPCK-treated, $40 \mathrm{U} / \mathrm{mg}$ ) were purchased from E. Merck Inc. (Darmstadt, Germany). Soybean TI was purchased from Roche Applied Science (Mannheim, Germany). Dithiothreitol, EDTA.2Na, electrophoretic reagents, human LDL, and other chemicals and reagents were purchased from Sigma Chemical Co. (St. Louis, MO, USA). Thiolyte ${ }^{\circledR}$ (monobromobimane reagent, $\mathrm{mBBr}$, cat. 596105) was from Millipore Co. (MA, USA). The prestained protein marker for SDS-PAGE was from Bioman Sci. Co. LTD (Taiwan). The disposable PD-10 desalting column and $\mathrm{CNBr}$-activated Sepharose $4 \mathrm{~B}$ resins were purchased from Amersham Biosciences (Uppsala, Sweden).

\section{Plant materials and TI purification}

Fresh roots of SP (Ipomoea batatas (L.) Lam 'Tainong 57') were purchased from a supermarket. Extraction and purification processes were according to previous methods (Hou and Lin 1997a, b; Hou et al. 2001, 2004, 2005). The SP crude extracts were loaded to an affinity column of self-prepared trypsin-Sepharose $4 \mathrm{~B}$ resins, and the adsorbed TIs were eluted by changing $\mathrm{pH}$ value with $0.2 \mathrm{M} \mathrm{KCl}$ buffer ( $\mathrm{pH} 2.0$ ), the absorbed SPTIs proteins were collected, adjusted to $\mathrm{pH} 7.5$, dialyzed against distilled water, and then lyophilized for further uses. 
Protections against $\mathrm{Cu}^{2+}$-mediated LDL oxidation by SPTIs The capacity of purified SPTIs to prevent $\mathrm{Cu}^{2+}$-mediated human LDL oxidation was measured by TBARS assay using absorbance at $532 \mathrm{~nm}$ following the previous method (Hou et al. 2005). After dialysis against $10 \mathrm{mM}$ phosphate buffer ( $\mathrm{pH}$ 7.4) overnight to remove EDTA, the total $100 \mu \mathrm{l}$ solution contained $20 \mu \mathrm{l}$ of dialyzed LDL $(2 \mathrm{mg} / \mathrm{ml}), 40 \mu \mathrm{l}$ of SPTIs $(1 \mathrm{mg} / \mathrm{ml})$, and tenfold-diluted PBS containing $10 \mu \mathrm{l}$ of $1 \mathrm{mM}$ copper (II) sulfate at $37^{\circ} \mathrm{C}$ for $24 \mathrm{~h}$. The tenfold-diluted PBS instead of SPTIs solution was used in the control. During reaction time interval of 0.5-, 1-2-, 3-, 4-, 6-, and 24-h, the $\mathrm{Cu}^{2+-}$ mediated reaction was stopped by adding $10 \mu \mathrm{l}$ of $1 \mathrm{mM}$ EDTA solution, and then for TBARS assay. The EDTA was added before copper (II) sulfate in the LDL/SPTIs mixture was used for the zero time assay.

\section{SPTIs bound to $\mathrm{LDL}$ during $\mathrm{Cu}^{2+}$-mediated $\mathrm{LDL}$ oxidation} The dialyzed LDL $(10 \mu \mathrm{l}, 5.8 \mu \mathrm{g})$ mixed with SPTIs $(20 \mu \mathrm{l}$, $20 \mu \mathrm{g}$ ), copper (II) sulfate $(5 \mu \mathrm{l}, 100 \mu \mathrm{M}$ in the final concentration), and tenfold-diluted PBS $(15 \mu \mathrm{l})$ were reacted at $37^{\circ} \mathrm{C}$ for $0,0.5,1,2,4,8$, and $24 \mathrm{~h}$. The dialyzed LDL mixed with copper (II) sulfate performed in the parallel experiment. The reaction was stopped by adding $5 \mu \mathrm{l}$ of $1 \mathrm{mM}$ EDTA solution. The SPTIs bound LDL during $\mathrm{Cu}^{2+}$-mediated LDL oxidation was carried out on a discontinuous Tris-glycine native polyacrylamide gel electrophoresis (PAGE) gel system, one $4 \%$ stacking gel and two layers of separation gel of $5 \%$ gel $(4 \mathrm{~cm})$ and $12.5 \%$ gel $(2.5 \mathrm{~cm})$. After electrophoresis, the native gel was fixed with $12.5 \%$ trichloroacetic acid for at least $30 \mathrm{~min}$, and stained by Comassie brilliant blue R-250 for proteins. The images in the selected square frame of stained proteins were quantified by a Syngene G:bBOX imaging system (Syngene, UK).

\section{SPTIs bound to oxLDL revealed by native PAGE gels and HPLC}

The dialyzed LDL was reacted with copper (II) sulfate at $37^{\circ} \mathrm{C}$ for $24 \mathrm{~h}$, and the reaction was terminated by adding EDTA solution (the final concentration of $100 \mu \mathrm{M}$ ) as the oxLDL. The solution was stored at $4{ }^{\circ} \mathrm{C}$ for further uses. The different amounts of SPTIs $(1,5,10$, and $15 \mu \mathrm{g})$ was mixed with oxLDL (about $10 \mu \mathrm{g}$ ) in tenfold-diluted PBS at $37{ }^{\circ} \mathrm{C}$ for $30 \mathrm{~min}$. The SPTIs bound oxLDL was carried out on a discontinuous Tris-glycine native PAGE gel system, one $4 \%$ stacking gel and two layers of separation gel of $4 \%$ gel $(4 \mathrm{~cm})$ and $20 \%$ gel $(2.5 \mathrm{~cm})$. After electrophoresis, the gel was placed in $12.5 \%$ trichloroacetic acid solution for $30 \mathrm{~min}$, and stained proteins by Comassie brilliant blue R-250. The images in the selected square frame of stained proteins were quantified by a
Syngene G:bBOX imaging system (Syngene, UK). For HPLC analysis, the total $300 \mu \mathrm{l}$ mixture contained SPTIs $(120 \mu \mathrm{g})$ and oxLDL $(120 \mu \mathrm{g})$ in tenfold-diluted PBS for $1 \mathrm{~h}$. The SPTIs/oxLDL solution was rapidly mixed either with equal volume of distilled water or glycine- $\mathrm{HCl}$ buffer ( $\mathrm{pH}$ 2.0), and the molecular size shift was analyzed by a TSKgel G3000PW $\mathrm{XL}_{\mathrm{XL}}(7.8 \times 300 \mathrm{~mm})$ gel filtration column in the Breeze HPLC system. The SPTIs alone in glycine- $\mathrm{HCl}$ buffer ( $\mathrm{pH}$ 2.0) was used for comparisons. The Breeze HPLC system (Waters Co., MA, USA) was equipped with pump (Waters 1525) and UV detector (Water 2487). The $10 \mathrm{mM}$ Tris- $\mathrm{HCl}$ buffer (pH 7.9) containing $0.1 \mathrm{M} \mathrm{NaCl}$ was used as the mobile phase with flow rate of $0.5 \mathrm{ml} / \mathrm{min}$. Each $20 \mu \mathrm{l}$ sample was injected and the absorbance at $280 \mathrm{~nm}$ was monitored.

\section{LDL or oxLDL bound onto self-prepared SPTIs-Sepharose 4B affinity column}

The preparation of SPTIs-Sepharose 4B affinity column was according to the previous method (Hou and Lin 2002) using CNBr-activated Sepharose $4 B$ resins. About $300 \mu \mathrm{g}$ LDL or oxLDL in tenfold-diluted PBS were loaded onto the SPTIs-Sepharose 4B affinity column. After being washed with tenfold-diluted PBS for 15 fractions and $10 \mathrm{mM}$ Tris- $\mathrm{HCl}$ buffer ( $\mathrm{pH} 7.9)$ containing $100 \mathrm{mM} \mathrm{NaCl}$ for another 15 fractions, the bound ones were eluted by $0.2 \mathrm{M} \mathrm{KCl}(\mathrm{pH} \mathrm{2.0)}$. Each fraction was monitored the absorbance at $280 \mathrm{~nm}$. The flow rate was $30 \mathrm{ml} / \mathrm{h}$ and each tube contained $5.0 \mathrm{ml}$. The unbound fractions in tenfold-diluted PBS washing were designed as fraction 1 , and the bound fractions eluted by $0.2 \mathrm{M}$ $\mathrm{KCl}$ buffer ( $\mathrm{pH} 2.0$ ) were saved, adjusted to 7.4, as fraction 2. Each fraction was concentrated with centriprep 10 to small volumes to perform native gel electrophoresis.

\section{Reductions and free thiol-labeling of SPTIs and soybean Tls, activity stains in SDS-PAGE gels, and LDL or oxLDL bindings}

The reductions of SPTIs or soybean TIs by dithiothreitol (DTT) were freshly prepared according to the previous methods (Trümper et al. 1994). The equal volume of purified SPTIs and soybean TI at $2 \mathrm{mg} / \mathrm{ml}$ were mixed with $20 \mathrm{mM}$ DTT in tenfold-diluted PBS for $2 \mathrm{~h}$, and the excess of DTT was removed by the disposable PD-10 column balanced with distilled water. The free thiollabeling in native or reduced SPTIs or commercial soybean TIs were achieved by thiolyte ${ }^{\circledR}$ fluorescent dyes following the previous methods (Kobrehel et al. 1991; Hou and Lin 1997a), and then performed electrophoresis. After electrophoresis, the gel was stained for proteins by Comassie brilliant blue R-250; for TI activity stains, the SDS was removal by $25 \%$ isopropanol in $10 \mathrm{mM}$ Tris buffer ( $\mathrm{pH}$ 7.9), and then the gel was placed in trypsin 
solution at $37{ }^{\circ} \mathrm{C}$ for $30 \mathrm{~min}$ for gentle shakings. The gel was stained in the $80 \mathrm{ml}$ substrate-dye solution containing $20 \mathrm{mg} N$-acetyl-phenylalanine $\beta$-naphthyl ester and $40 \mathrm{mg}$ tetrazotized $O$-dianisidine under light protections at $37^{\circ} \mathrm{C}$ for $30 \mathrm{~min}$ (Hou and Lin 1997a, b), and the blank zones against deep purple background showed TI positions. For comparing different binding capacities of SPTIs, reduced SPTIs, soybean TIs, and reduced soybean TIs toward LDL and oxLDL, the native or reduced TIs $(10 \mu \mathrm{g})$ and LDL or oxLDL $(10 \mu \mathrm{g})$ in tenfold-diluted PBS were mixed for $1 \mathrm{~h}$, and then were carried out on a discontinuous Tris-glycine native PAGE gel system as above-mentioned.

\section{Statistical analysis}

Data were expressed as mean $\pm \mathrm{SD}$. Multiple group comparisons were performed using one-way analysis of variance (ANOVA) and the post hoc Tukey's test, and the different uppercase alphabet in each treatment or lowercase alphabet in each treatment were considered significantly different $(P<0.05)$. The student's t-test was used for two group comparisons of binding capacities between LDL and (LDL + native or reduced forms of SPTIs or soybean TIs), or oxLDL and (oxLDL + native or reduced forms of SPTIs or soybean TIs). A difference was considered statistically significant when $P<0.05\left(^{*}\right)$ or $P<0.01$ ${ }^{(* *)}$ or $P<0.001\left(^{(* * *)}\right.$. The GraphPad Prism 6 (San Diego, CA) was used for statistical analysis.

\section{Results}

Effects of SPTIs on $\mathrm{Cu}^{2+}$-mediated LDL oxidation and SPTIs bound to oxLDL

Figure 1a showed results of the $\mathrm{Cu}^{2+}$-mediated LDL oxidations during 24-h (expressed as TBARS formation, A532 nm) without or with SPTIs additions. It was found that the LDL oxidation (TBARS formation) was growing rapidly, and 4-h reaction reached to the top and then gradually decreased. However, the parallel experiment of LDL oxidations in the presence of SPTIs showed to reduce $\mathrm{Cu}^{2+}$-mediated LDL oxidations in the first 6-h intervals and then gradually closed to the control. Figure $1 \mathrm{~b}$ showed the protein stains in the native PAGE gel of the fixed ratio of LDL/SPTIs $(5.8 \mu \mathrm{g} / 20 \mu \mathrm{g}$, W/W) or LDL only $(5.8 \mu \mathrm{g})$ at $\mathrm{Cu}^{2+}$-mediated LDL oxidations during $0,0.5,1,2,4,8$, and 24-h intervals, and each band intensity in the selected square frame was quantified. The $\mathrm{Cu}^{2+}$-mediated LDL oxidations (lanes 3, 5, 7, 9, 11, and $13)$ could significantly increase intensity of protein stains $(P<0.05)$ in the LDL position compared those in the nonoxidized LDL (zero time, lane 1 ). The 0.5 -h to 2 -h LDL oxidations reached the highest band intensities (lanes 3, 5 , and 7), and then gradually decreased (lanes 9, 11, and 13 , respectively for $4-h, 8-h$, and $24-h$ ), which showed no significant difference $(P>0.05)$ compared to that in the zero time (lane 1).

While, SPTIs additions in $\mathrm{Cu}^{2+}$-mediated LDL oxidations (lanes 4, 6, 8,10,12, and 14), the intensities of protein bands in the LDL position at each time interval of 0.5-, 1-, 2-, 4-, 8-, and 24-h reactions were enhanced compared to SPTIs additions without LDL oxidation (lane 2, zero time). It was noted that non-oxidized LDL in the presence of SPTIs (lane 2) showed higher intensity of protein stains in the LDL position (assumed as SPTIs/ LDL complex) compared to that in the non-oxidized LDL only (lane 1), which showed SPTIs might bind to nonoxidized LDL to form SPTIs/LDL complex (lane 2). The $0.5-\mathrm{h}$ and 1-h LDL oxidations in the presence of SPTIs reached the highest band intensity (lanes 4 and 6) and then gradually decreased (lanes $8,10,12$, and 14 , respectively for 2-h, 4-h, 8-h, and 24-h). The intensity of protein stains in 24-h reaction showed no significant difference $(P>0.05)$ compared to that in the zero time in the presence of SPTIs (lane 2), but showed higher intensities compared to the non-oxidized LDL only (lane 1).

\section{SPTIs bound to oxLDL revealed by native PAGE gels and HPLC}

The dialyzed LDL was reacted with copper (II) sulfate at $37^{\circ} \mathrm{C}$ for $24 \mathrm{~h}$, and the reaction was terminated by adding EDTA solution as the oxLDL. The SPTIs were mixed with oxLDL in tenfold-diluted PBS at $37{ }^{\circ} \mathrm{C}$ for $30 \mathrm{~min}$ and then performed native electrophoresis. Figure 2a showed the protein stains in the native PAGE gel of the different ratios of fixed amounts of oxLDL $(10 \mu \mathrm{g})$ with different amounts of SPTIs $(1,5,10$, and $15 \mu \mathrm{g})$. The SPTIs only $(1,5,10$, and $15 \mu \mathrm{g})$ or LDL only $(10 \mu \mathrm{g})$ were used as the controls, and the band intensity in the selected square frame was quantified. The addition of SPTIs at dose of $1,5,10$, and $15 \mu \mathrm{g}$, seemed to enhance protein stains of oxLDL band, but only SPTIs addition at doses of 1,5 , and $10 \mu \mathrm{g}$ showed significant differences compared to that of oxLDL only $(P<0.05)$.

The HPLC equipped with gel filtration column was used to analyze the molecular size changes of SPTIs bound oxLDL (Fig. 2b). There were two conditions for SPTIs/oxLDL binding assays, which the reaction was in the distilled water (red line of gel filtration chromatogram) or in the glycine- $\mathrm{HCl}$ buffer ( $\mathrm{pH}$ 2.0) (green line of gel filtration chromatogram). The main peak of high molecular size of SPTIs/oxLDL complex was around $12 \mathrm{~min}$ in distilled water (red line). While in the glycine- $\mathrm{HCl}$ buffer ( $\mathrm{pH} 2.0$, green line), the amounts of original high molecular size (assumed as SPTIs/oxLDL complex) was reduced and shifted to smaller molecular sizes at $14 \mathrm{~min}$ and $18 \mathrm{~min}$ of HPLC chromatogram, which was similar to positions of SPTIs in glycine- $\mathrm{HCl}$ 
$\mathbf{a}$

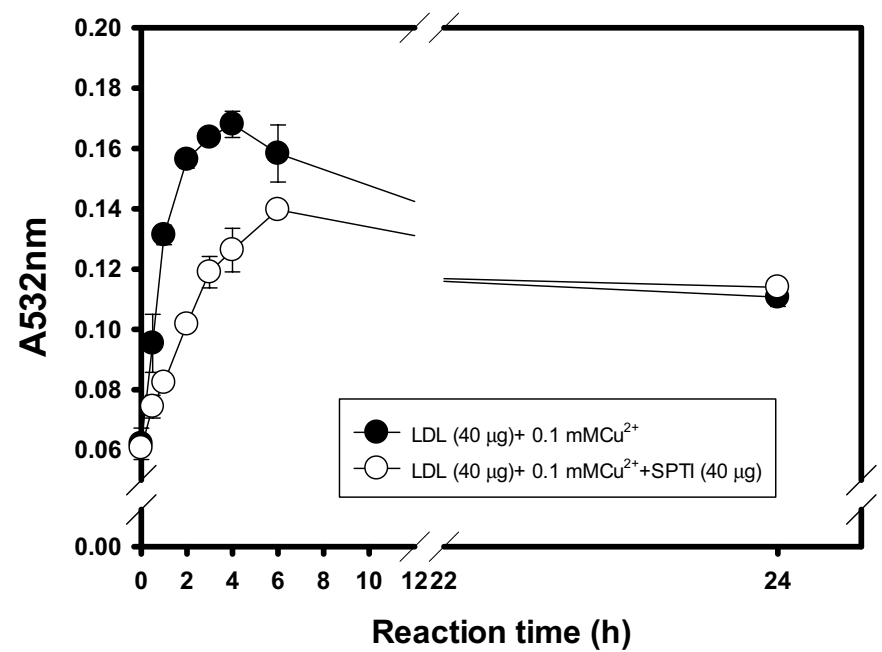

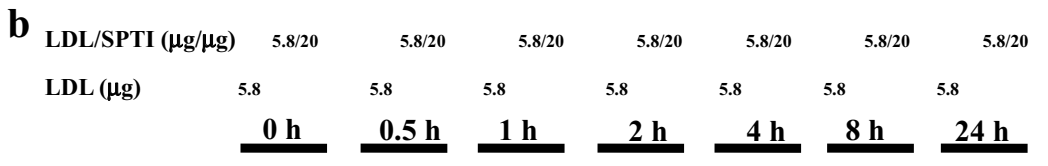

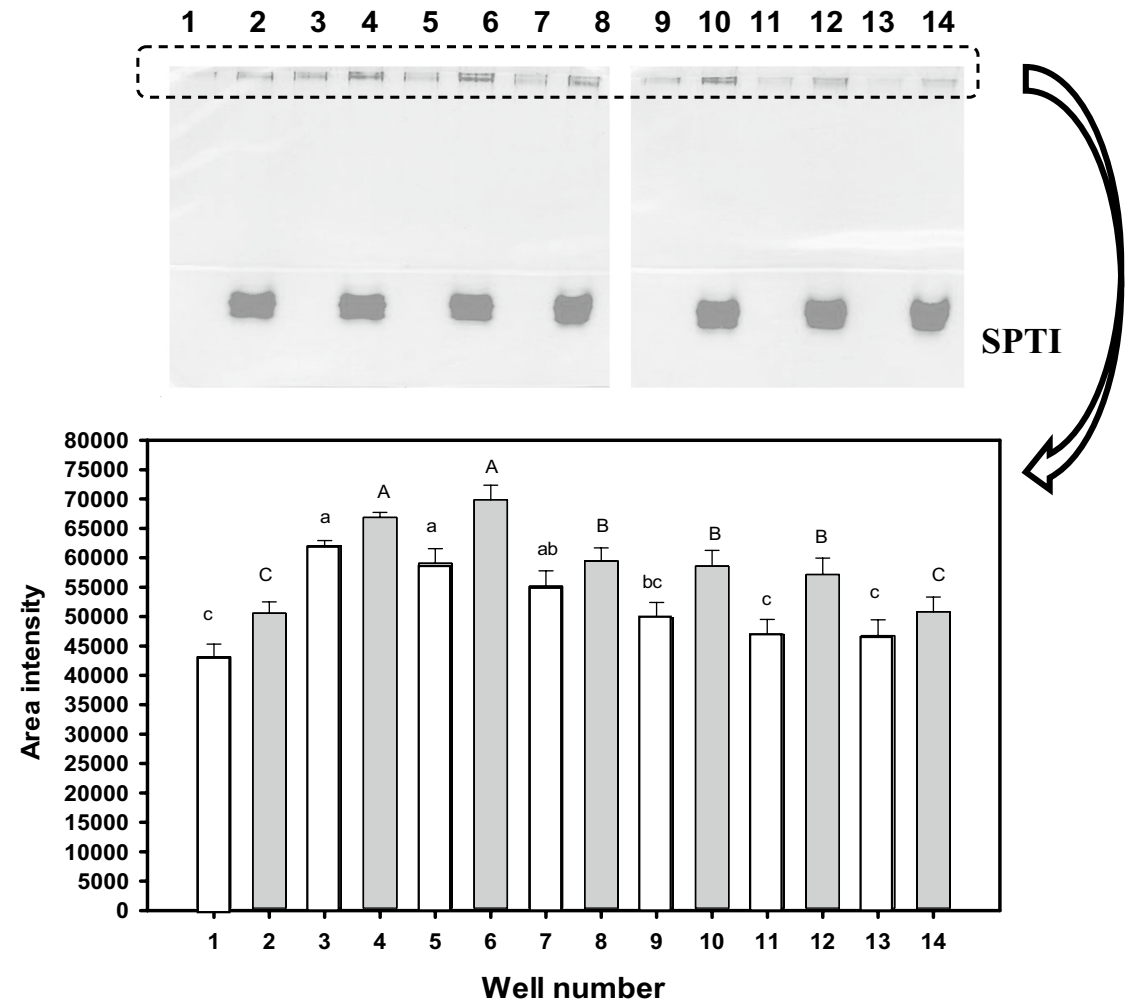

Fig. 1 a Effect of sweet potato trypsin inhibitors (SPTIs) on $0.1 \mathrm{mM} \mathrm{Cu}^{2+}$-mediated low-density lipoprotein (LDL) oxidation during 24-h reactions evaluated by TBARS methods, and $\mathbf{b}$ during $\mathrm{Cu}^{2+}$-mediated LDL oxidation, effects of SPTIs on LDL or oxLDL binding were evaluated by protein stains in native PAGE gels with two layers of separation gels. The selected square frame was quantified by a Syngene G:bBOX imaging system (Syngene, UK). Values were presented as mean \pm SD and were analyzed using one-way ANOVA, followed by a post hoc Tukey's test for multiple comparisons. The different marked symbols (LDL only, lowercase; LDL/SPTIs model, uppercase) in each bar were significantly different $(P<0.05)$ 

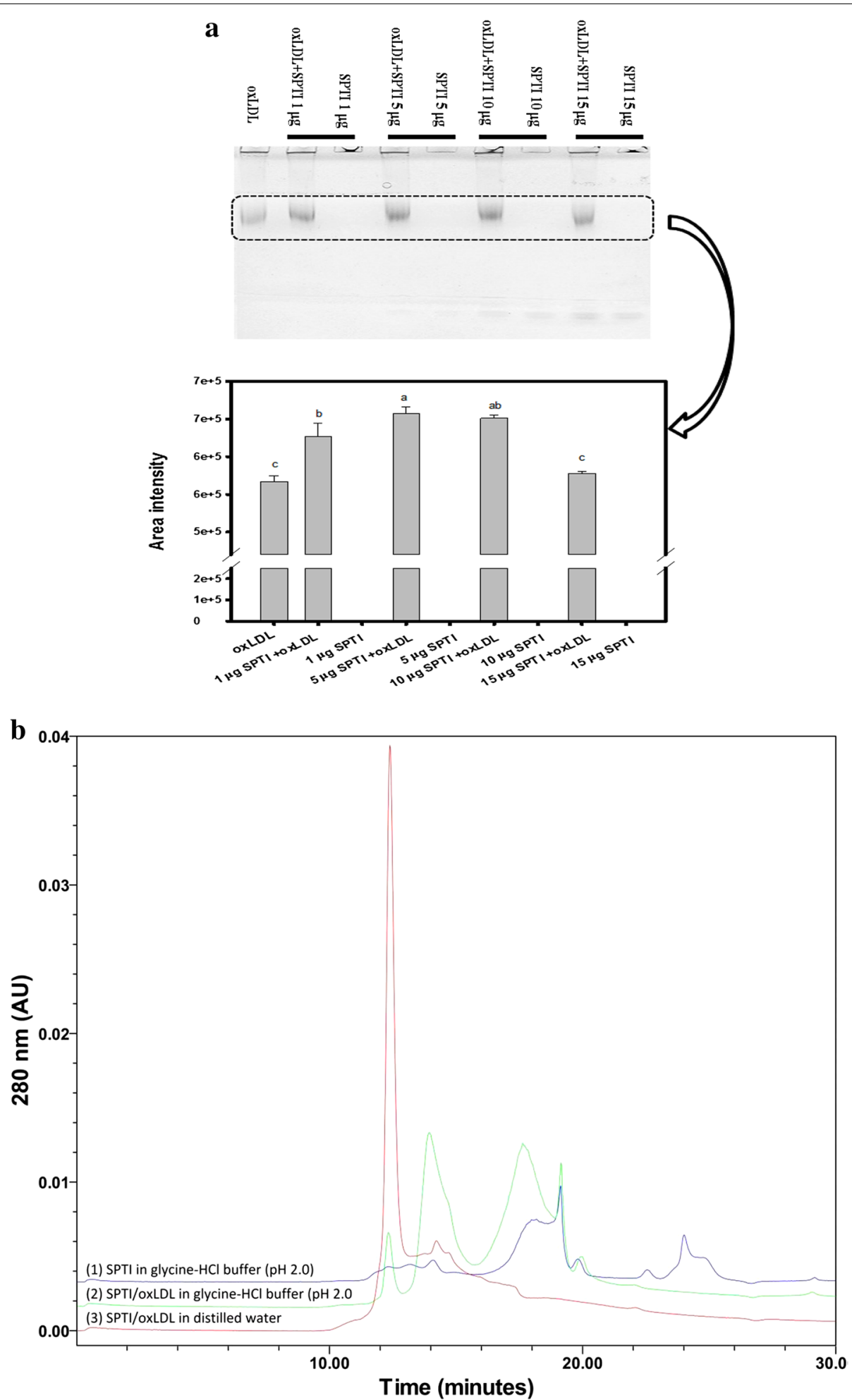

Fig. 2 a Effects of SPTIS on oxLDL binding were evaluated by protein stains in native PAGE gels with two layers of separation gels. The selected square frame was quantified by a Syngene G:bBOX imaging system (Syngene, UK), and b the HPLC equipped with TSKgel G3000PW $(7.8 \times 300 \mathrm{~mm})$ gel filtration column was used to analyze the molecular size changes of SPTIs/OxLDL complex. (1) The SPTIs alone in glycine-HCl buffer ( $\mathrm{pH}$ 2.0, deep blue line); (2) SPTIs/oxLDL binding assays in the glycine-HCl buffer (pH 2.0, green line); (3) SPTIs/oxLDL binding assays in the distilled water (red line). Values were presented as mean \pm SD and were analyzed using one-way ANOVA, followed by a post hoc Tukey's test for multiple comparisons. The different marked symbols in each bar were significantly different $(P<0.05)$ 
buffer ( $\mathrm{pH}$ 2.0, purple line). Based on the results of Fig. 2, the SPTI could directly bind to oxLDL and low $\mathrm{pH}$ condition $(\mathrm{pH} 2.0)$ might break the interactions.

\section{LDL or oxLDL bound onto self-prepared SPTIs-Sepharose $4 \mathrm{~B}$ affinity column}

Figure 3a showed chromatogram of LDL or oxLDL on a SPTIs-Sepharose affinity column. Fraction 1 (Fra.1) was the unbound portion of LDL or oxLDL washed by tenfold-diluted PBS, and the fraction 2 (Fra.2) was the bound portion of LDL or oxLDL eluted by $0.2 \mathrm{M} \mathrm{KCl}(\mathrm{pH} 2.0)$. It was found that some portions of LDL or oxLDL could be eluted from the SPTIs-affinity column by lowering $\mathrm{pH}$ to 2.0. Figure $3 \mathrm{~b}$ showed the protein stains in the native PAGE gel of LDL-Fra1 and oxLDL-Fra1 or LDL-Fra2 and oxLDL-Fra2. The bound fraction (Fra. 2, arrow indicated)

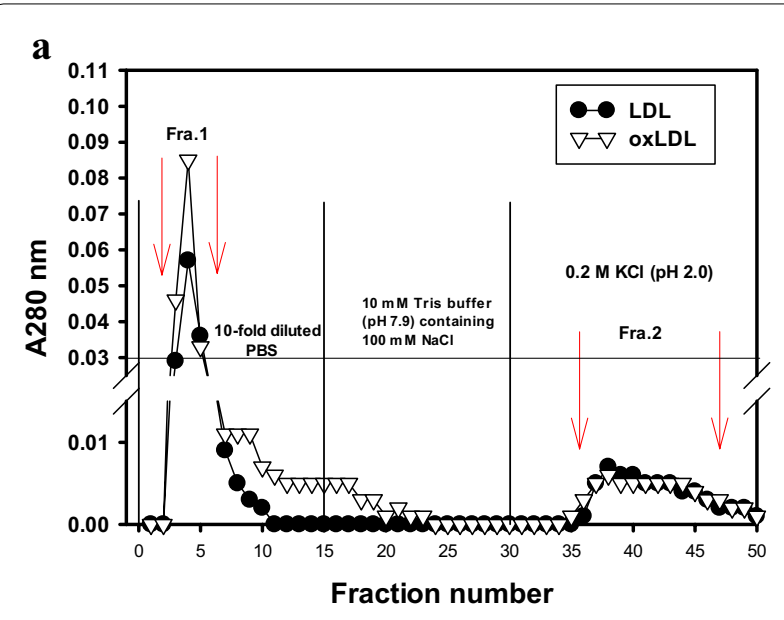

b

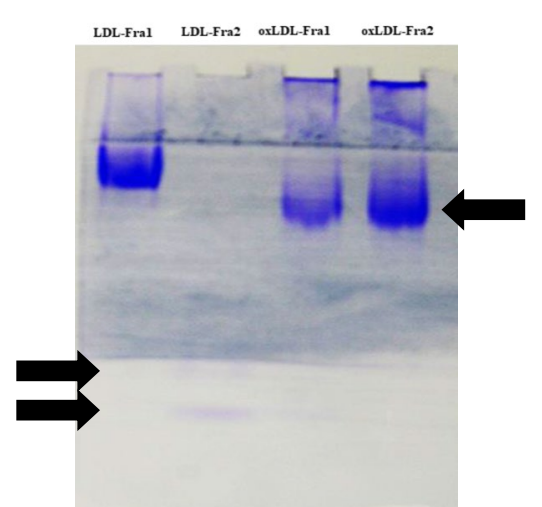

Fig. 3 a Chromatograms of LDL or oxLDL on a SPTIs-Sepharose affinity column. Fraction 1 (Fra.1) was the unbound portion of LDL or oxLDL washed by tenfold-diluted PBS, and the fraction 2 (Fra.2) was the bound portion of LDL or oxLDL eluted by $0.2 \mathrm{M} \mathrm{KCl}(\mathrm{pH} 2.0)$, and $\mathbf{b}$ the protein stains in the native PAGE gel with two layers of separation gels for LDL-Fra1, oxLDL-Fra1, LDL-Fra2, and oxLDL-Fra2. Arrows indicated the unidentified components from eluents of LDL and oxLDL showed some different protein stains in the native PAGE gel. These results confirmed LDL or oxLDL could bind onto SPTIs, and interactions could be broken by lowering $\mathrm{pH}$ value to 2.0 .

\section{Effects of native or reduced SPTIs and soybean TIs on LDL or oxLDL binding capacities}

Figure 4a showed the protein stains (panel A), activity stains (panel B), and stains of free thiol-labeling (panel C). The lane 1 and lane 2 , respectively, were native and reduced SPTIs; the lane 3 and lane 4, respectively, were native and reduced soybean TIs. From the results of panel C of Fig. 4a, the reduced SPTIs (lane 2) and the reduced soybean TIs (lane 4) showed more intense thiollabeling stains compared to each native proteins. The native or reduced forms of SPTIs and soybean TIs all showed trypsin inhibitory activities (panel B, Fig. 4a), and the main proteins of SPTIs (around $25 \mathrm{kDa}$ ) showed higher molecular size compared to that of soybean TIs (around $20 \mathrm{kDa}$ ) in the SDS-PAGE gels (panel A, Fig. 4a). These native and reduced forms of SPTIs and soybean TIs were used to test LDL and oxLDL binding capacities by the protein stains in the native PAGE gel (Fig. 4b), and the selected square frame was quantified by a Syngene G:bBOX imaging system (Syngene, UK). The lanes 1 to 5 (Fig. 4b), respectively, were LDL only, LDL/SPTI, LDL/reduced SPTI, LDL/soybean TI, and LDL/reduced soybean TI. The lanes 6 to 10 (Fig. 4b), respectively, were oxLDL only, oxLDL/SPTIs, oxLDL/reduced SPTIs, oxLDL/soybean TIs, and oxLDL/reduced soybean TIs. Under the same binding conditions, it was found that the native and reduced forms of SPTIs and soybean TIs showed LDL and oxLDL binding capacities and showed significant differences compared to the LDL or oxLDL only $(P<0.05,0.01,0.001)$. It was noted that the soybean TIs (native or reduced forms) showed higher average oxLDL binding capacities than those of SPTIs under the same amounts used (lanes 7 to 10) based on selected square frame quantifications. It was found that the soybean TIs (native or reduced forms) showed higher oxLDL binding capacities (lanes 9 and 10) than those of LDL (lanes 4 and 5) under the same amounts used. The native SPTIs showed similar binding capacities toward LDL and oxLDL (lanes 2 and 7), and reduced SPTIs also showed the similar results (lanes 3 and 8).

\section{Discussion}

The present results showed that the SPTIs exhibited LDL binding capacities and oxLDL binding capacities, which might be related to lower TRARS levels during $\mathrm{Cu}^{2+}$-mediated LDL oxidations. The reduced SPTIs, soybean TI, and reduced soybean TI also showed LDL and oxLDL binding capacities. Though SPTIs (or sporamin) 
(See figure on next page.)

Fig. 4 a Protein stains (panel A), activity stains (panel B), and free thiol-labeling stains (panel C). The lane 1 and lane 2, respectively, were native and reduced SPTIs; the lane 3 and lane 4, respectively, were native and reduced soybean TIs, and $\mathbf{b}$ effects of the native and reduced forms of SPTIS and soybean TIs on LDL and oxLDL binding capacities evaluated by the protein stains in the native PAGE gel with two layers of separation gels, and the selected square frame was quantified by a Syngene G:bBOX imaging system (Syngene, UK). Each of LDL, oxLDL, SPTIs, reduced SPTIs, soybean TIs, and reduced soybean Tls were $10 \mu \mathrm{g}$ in the mixture. The lanes 1 to 5, respectively, were LDL only, LDL/SPTIs, LDL/reduced SPTIs, LDL/soybean TIs, and LDL/reduced soybean TIs. The lanes 6 to 10, respectively, were oxLDL only, oxLDL/SPTIs, oxLDL/reduced SPTIs, oxLDL/soybean TIs, and oxLDL/reduced soybean TIs. Data were expressed as mean \pm SD. The student's t-test was used for two group comparisons of binding capacities between $L D L$ and ( $L D L+$ native or reduced forms of SPTIs or soybean TIs), or oxLDL and (oxLDL + native or reduced forms of SPTIs or soybean TIs). A difference was considered statistically significant when $P<0.05\left({ }^{*}\right)$ or $P<0.01(* *)$ or $P<0.001(* * *)$

and soybean TI used in the present study were belonged to the Kunitz-type trypsin inhibitors, both amino acid sequences shared only 30\% identity and 50\% similarity (Senthilkumar and Yeh 2012). It was reported that the purified NADPH/thioredoxin system could facilitate SPTIs reductions in vitro and might beneficial as nitrogen sources for SP sprouting in vivo (Huang et al. 2004). The purified SPTIs showed thiol-disulfide interchanges to regenerate ascorbate from dehydroascorbate (as dehydroascorbate reductase activity), and the free thiol labeling stains of SPTIs were reported (Hou and Lin 1997a). Therefore, parts of purified SPTIs might exhibit free thiol stains in nature (Fig. 4a, lane 1 of panel C). It was reported SPTIs exhibited free radical scavenging activities, including DPPH radicals and hydroxyl radicals (Hou et al. 2001, 2005), and the scavenging activities were contributed by Cys residues for the former and by Trp residues for the latter (Hou et al. 2005). The higher concentrations of SPTIs $(12,15$, and $18 \mathrm{mg} / \mathrm{ml}$ purified from TN57 SP cultivar; 4,5 , and $6 \mathrm{mg} / \mathrm{ml}$ from TN65 SP cultivar) were used significantly to reduce $\mathrm{Cu}^{2+}$-mediated LDL oxidation, which was catalyzed by $10 \mu \mathrm{M} \mathrm{Cu}^{2+}$ at $37^{\circ} \mathrm{C}$ and determined by TBARS methods at the fixed 24-h reaction (Hou et al. 2005). The present study showed that no protective activity of SPTIs against LDL oxidation was found at fixed 24-h reaction, however, in the first 6-h SPTIs additions showed to reduce LDL oxidations and also formed LDL/SPTIs or oxLDL/SPTIs complex.

The chemical compositions of LDL particle comprised a glycoprotein of apoprotein B-100 (apoB-100), regulatory proteins, a monolayer of phospholipid, and a hydrophobic core, which totally accounted for about $75 \%$ total LDL weights (Alipov et al. 2017). The activated monocytes, macrophages, and endothelial cells in vivo could produce reactive oxygen species, lipoxygenase, and peroxidase, along with trace metal ions $\left(\mathrm{Fe}^{3+}\right.$ and $\left.\mathrm{Cu}^{2+}\right)$ to enhance LDL oxidations. The processes of LDL oxidation generated fatty acid oxidation products, lipid-derived products and lipid-protein adducts, and protein crosslinking products via oxidations (Parthasarathy et al. 2010; Alipov et al. 2017). The 4-hydroxy-2-nonenal (HNE), one of reactive lipid products, was identified by LC/MS/MS as the lipid-protein adducts in histidine residue of the apoB-100 tryptic hydrolysates in $\mathrm{Cu}^{2+}$-mediated $\mathrm{LDL}$ oxidation (Bolgar et al. 1996). The oxLDL, but not LDL, could bind receptors to enhance oxLDL uptakes and lipid accumulations in subendothelial intima to form the foam cells and the advanced plaque, which the levels of oxLDL were used as a biomarker of endothelial dysfunction (Alipov et al. 2017).

The human LDL (or apoB-100) formed complex in vivo with endogenous oxidized $\alpha 1$-antitrypsin (oxidized AT) isolated from LDL fraction by the oxidized AT antibodycoupled affinity column, and LDL/oxidized AT complex was also found in atherosclerotic lesions and the arterial wall (Mashiba et al. 2001). The secretory AT, belonged to the serpin family to regulate serine proteinase, was produced mainly by liver or lipopolysaccharide-stimulated macrophages. The main function of AT was showed irreversibly to bind and inactivate neutrophil elastase or to inhibit excess proteinase activities in the inflammatory sites (Knoell et al. 1998). However, the oxidized AT lost the inhibitory activity against proteinases by oxidizing methionine residue to methionine sulfoxide in the active site by peroxide, peroxynitrite, or hydroxyl radicals attacks (Mashiba et al. 2001). The LDL and oxLDL could bind onto SPTIs-affinity column (Fig. 3) and some unidentified components were eluted by $0.2 \mathrm{M} \mathrm{KCl}-\mathrm{HCl}$ buffer ( $\mathrm{pH}$ 2.0). It was not clear the bound components from LDL or oxLDL onto SPTIs-affinity column. It will be possible to isolate and identify the eluents (or binding components) from the SPTIs-affinity column by LC/MS/ MS techniques.

The Asp-hemolysin was a hemolytic toxin and produced by Aspergillus fumigatus, which was reported to form complex with oxLDL in $\mathrm{Cu}^{2+}$-mediated LDL oxidation during 24-h reactions, and 4- $\mathrm{C} \mathrm{Cu}^{2+}$-mediated LDL oxidation showed the highest bindings (Kudo et al. 2001, 2002). The Asp-hemolysin also formed complex with LDL to the lesser extent (Kudo et al. 2001). The Asp-hemolysin showed to bind lysophosphatidylcholine (LysoPC) portion in oxLDL particles (Kudo et al. 2002). The synthesized peptides derived from Asp-hemolysin,

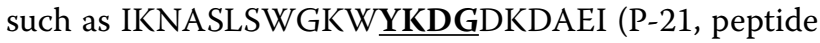


a

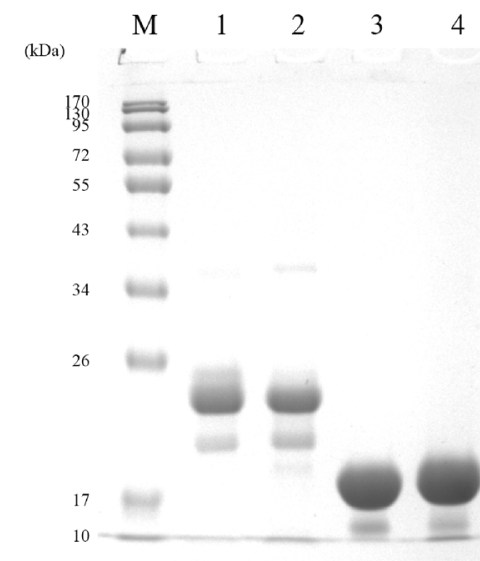

A

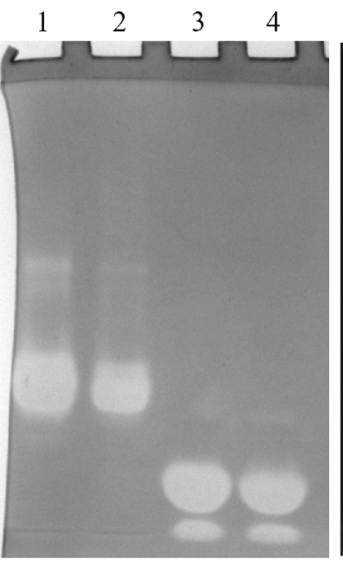

B

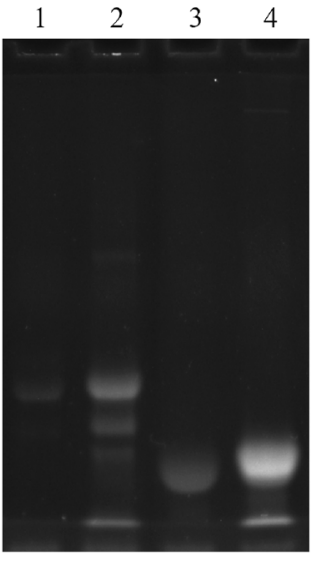

C

b

LDL oxLDL

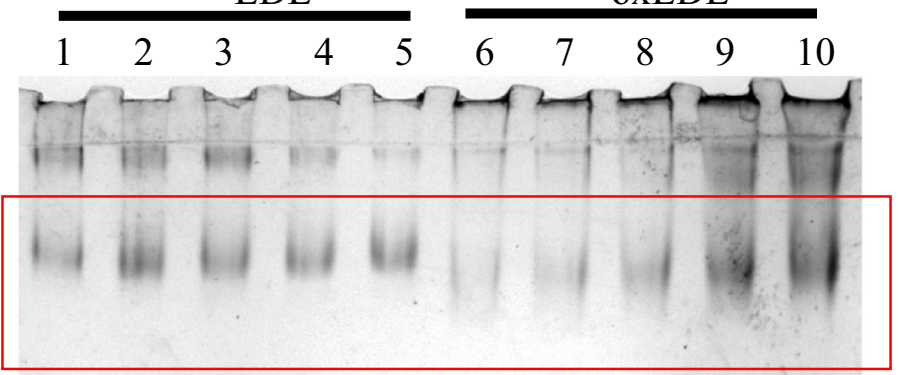

홓ㅎㅎ

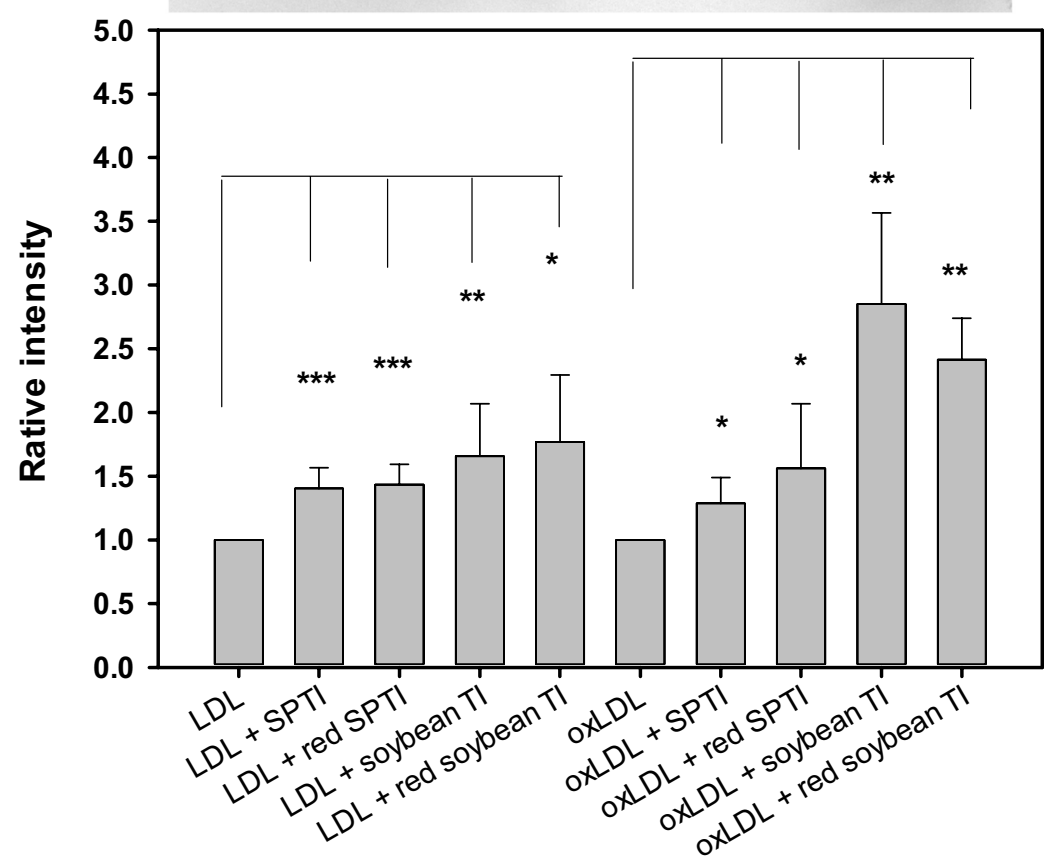


contained 21 amino acids) and YKDG (P-4, peptide contained 4 amino acids), showed oxLDL and LysoPC binding capacities, which the YKDG moiety in Asp-hemolysin might be the important region for oxLDL or LysoPC bindings (Kumagai et al. 2006). Based on the present results, it will be possible to synthesize biotinylated-peptides derived from peptic hydrolysis of SPTIs to evaluate the LDL and oxLDL bindings to identify binding regions in SPTIs for LDL and/or oxLDL.

\section{Conclusion}

In conclusion, native SPTIs showed to reduce copper ionmediated LDL oxidations. The native or reduced forms of SPTIs and soybean TIs showed LDL binding and oxLDL binding capacities in vitro. The free Cys residues and Trp residues in SPTIs (or in reduced forms of SPTIs), respectively, showed to contribute DPPH radical and hydroxyl radical scavenging activities (Hou et al. 2005), which might scavenge lipid peroxyl radicals induced by $\mathrm{Cu}^{2+}$ to break lipid chain reactions of LDL oxidations. The SPTIs might contain regions that could bind to apoB regions in LDL or oxLDL, which the LDL/SPTIs or oxLDL/SPTIs mixtures will investigate by anti-apoB antibody in native gels. Later, the SPTIs or its synthesized peptides will intervene in hypercholesterolemia animal models to evaluate the effects on LDL levels, which will investigate in the future.

\section{Acknowledgements}

Authors want to express thanks to the Jin-lung-yuan Foundation (2019 to 2020) for financial supports.

\section{Authors' contributions}

SYL, CJL, and WCH participated the discussion and concepts of experimental designs, MS writing and revision; YLL and WCH performed the analytical experiments. All authors read and approved the final manuscript.

\section{Funding}

No.

\section{Availability of data and materials}

All data generated during the study are interpreted in the manuscript.

\section{Ethics approval and consent to participate}

Not applicable.

\section{Consent for publication}

Not applicable.

\section{Competing interests}

The authors declare that they have no competing interests.

\footnotetext{
Author details

${ }^{1}$ School of Pharmacy, College of Pharmacy, Taipei Medical University, Taipei 110 , Taiwan. ${ }^{2}$ Ph.D. Program in Clinical Drug Development of Herbal Medicine, College of Pharmacy, Taipei Medical University, Taipei, Taiwan. ${ }^{3}$ Division of Gastroenterology, Department of Internal Medicine, Wan Fang Hospital, Taipei Medical University, Taipei, Taiwan. ${ }^{4}$ Department of General Medicine, School of Medicine, College of Medicine, Taipei Medical University, Taipei,
}

Taiwan. ${ }^{5}$ Graduate Institute of Pharmacognosy, Taipei Medical University, No. 250, Wu-Hsing Street, Taipei 110, Taiwan.

Received: 15 July 2020 Accepted: 18 September 2020

Published online: 24 September 2020

\section{References}

Alipov VI, Sukhorukov VN, Karagodin VP, Grechko AV, Orekhov AN (2017) Chemical composition of circulating native and desialylated low density lipoprotein: what is the difference? Vessel Plus 1:107-115

Bolgar MS, Yang CY, Gaskell SJ (1996) First direct evidence for lipid/protein conjugation in oxidized human low density lipoprotein. J Biol Chem 271:27999-28001

Food and Agriculture Organization of the United Nations (1999) Production yearbook. FAO Statistics, vol 53. FAO, Italy

Food and Agriculture Organization of the United Nations. Roots, tubers, plantains and bananas in human nutrition. 1990. FAO, Rome, Italy (http:// www.fao.org/3/t0207e/T0207E04.htm\#4.\%20Nutritive\%20value)

Gießauf A, Steiner E, Esterbauer H (1995) Early destruction of tryptophan residues of apolipoprotein $B$ is a vitamin $E$-independent process during copper-mediated oxidation of LDL. Biochim Biophys Acta 1256:221-232

Heuzé V, Tran G, Hassoun P, Renaudeau D, Bastianelli D (2015) Sweet potato (Ipomoea batatas) tubers (https://www.feedipedia.org/node/745)

Hou WC, Lin YH (1997a) Dehydroascorbate reductase and monodehydroascorbate reductase activities of trypsin inhibitors, the major sweet potato (Ipomoea batatas [L.] Lam) root storage protein. Plant Sci 128:151-158

Hou WC, Lin YH (1997b) Polyamine-bound trypsin inhibitors in sweet potato (Ipomoea batatas [L.] Lam cv. Tainong 570 storage roots, sprouted roots and sprouts. Plant Sci 126:11-19

Hou WC, Lin YH (2002) Sweet potato (Ipomoea batatas (L.) Lam) trypsin inhibitors, the major root storage proteins, inhibit one endogenous serine protease activity. Plant Sci 163:733-739

Hou WC, Chen YC, Chen HJ, Lin YH, Yang LL, Lee MH (2001) Antioxidant activities of trypsin inhibitor, a $33 \mathrm{kDa}$ root storage protein of sweet potato (Ipomoea batatas (L.) Lam cv. Tainong 57). J Agric Food Chem 49:2978-2981

Hou WC, Chen HJ, Han CH, Lin CY, Lin YH (2004) Glutathione peroxidase-like activity of $33 \mathrm{kDa}$ trypsin inhibitor from roots of sweet potato (Ipomoea batatas [L.] Lam Tainong 57). Plant Sci 166:1541-1546

Hou WC, Han CH, Chen HJ, Wen CL, Lin YH (2005) Storage proteins of two cultivars of sweet potato (Ipomoea batatas L.) and their protease hydrolysates exhibited antioxidant activity in vitro. Plant Sci 168:449-456

Huang DJ, Chen HJ, Hou WC, Chen TE, Lin YH (2004) In vitro reduction of trypsin inhibitor by purified NADPH/thioredoxin system from sprouts of sweet potato (Ipomoea batatas (L.) Lam.) storage roots. Plant Sci 166:435-441

Huang DJ, Hou WC, Chen HJ, Lin YH (2006) Sweet potato (Ipomoea batatas (L.) Lam. Tainong 57) storage root mucilage exhibited angiotensin converting enzyme inhibitory activity in vitro. Bot Stud 47:397-402

Huang GJ, Chang HY, Chen HJ, Lu TL, Chang YS, Sheu MJ, Lin YH (2008) Effects of trypsin inhibitor on plasma antioxidant activity and lipid levels in mice from sweet potato roots. J Sci Food Agric 88:2556-2562

Huang SS, Hung HJ, Chiu CS, Chen HJ, Lin SS, Lin YC, Chang HY, Huang GJ, Lin YH (2009) Sweet potato trypsin inhibitor with thioltransferase-like and glutathione S-transferase-like activities. Bot Stud 50:443-450

Ishiguro K, Kurata R, Shimada Y, Sameshima Y, Kume T (2016) Effects of a sweetpotato protein digest on lipid metabolism in mice administered a high-fat diet. Heliyon. 2:e00201. https://doi.org/10.1016/j.heliyon.2016. e00201

Kawamura M, Heinecke JW, Chait A (1994) Pathophysiological concentrations of glucose promote oxidative modification of low density lipoprotein by a superoxide-dependent pathway. J Clin Invest 94:771-778

Knoell DL, Ralston DR, Coulter KR, Wewers MD (1998) Alpha 1-antitrypsin and protease complexation is induced by lipopolysaccharide, interleukin-1 $\beta$, and tumor necrosis factor-a in monocytes. Am J Respir Crit Care Med 157:246-255

Knott HM, Baoutina A, Davies MJ, Dean RT (2002) Comparative time-courses of copper-ion-mediated protein and lipid oxidation in low-density lipoprotein. Arch Biochem Biophys 400:223-232 
Kobrehel K, Yee BC, Buchanan BB (1991) Role of the NADP/thioredoxin system in the reduction of -amylase and trypsin inhibitor proteins. J Biol Chem 266:16135-16140

Kotani K, Yamada T, Taniguchi N (2010) The association between adiponectin, HDL-cholesterol and \& a1-antitrypsin-LDL in female subjects without metabolic syndrome. Lipids Health Dis 9:147. https://doi. org/10.1186/1476-511X-9-147

Kudo Y, Fukuchi Y, Kumagai T, Ebina K, Yokota K (2001) Oxidized low-density lipoprotein-binding specificity of Asp-hemolysin from Aspergillus fumigatus. Biochem Biophys Acta 1568:183-188

Kudo Y, Ootani T, Kumagai T, Fukuchi Y, Ebina K, Yokota K (2002) A novel oxidized low-density lipoprotein-binding protein, Asp-hemolysin, recognizes lysophosphatidylcholine. Biol Pharm Bull 25:787-790

Kumagai T, Tsutsumi H, Ogawa N, Naito S, Ebina K, Yokota K, Nagata K (2006) Oxidized low-density lipoprotein-binding specificity of the Asp-hemolysin-related synthetic peptides from Aspergillus fumigatus. Biol Pharm Bull 29:2181-2186

Lin YH (1988) Trypsin inhibitor activities as useful taxonomic traits of sweetpotato varieties. Euphytica 38:39-47

Lin YH, Chen HL (1980) Level and heat stability of trypsin inhibitor activity among sweet potato (Ipomoea batatas Lam.) varieties. Bot Bull Acad Sin $21: 1-13$

Maeshima M, Sasaki T, Asahi T (1985) Characterization of major proteins in sweet potato tuberous roots. Phytochem 24:1899-1902

Mashiba S, Wada Y, Takeya M, Sugiyama A, Hamakubo T, Nakamura A, Noguchi N, Niki E, Izumi A, Kobayashi M, Uchida K, Kodama T (2001) In vivo complex formation of oxidized \& aB1;1-antitrypsin and LDL. Arterioscler Thromb Vasc Biol 21:1801-1808

Mohanraj R, Sivasankar S (2014) Sweet potato (Ipomoea batatas [L.] Lam)-a valuable medicinal food: a review. J Med Food 17:733-741
Nakano E, Williamson MP, Williams NH, Powers HJ (2004) Copper-mediated $\mathrm{LDL}$ oxidation by homocysteine and related compounds depends largely on copper ligation. Biochim Biophys Acta 1688:33-42

Parthasarathy S, Raghavamenon A, Garelnabi MO, Santanam N (2010) Oxidized low-density lipoprotein. Methods Mol Biol 610:403-417. https://doi. org/10.1007/978-1-60327-029-8_24

Schnitzer E, Pinchuk I, Bor A, Fainaru M, Lichtenberg D (1997) The effect of albumin on copper-induced LDL oxidation. Biochim Biophys Acta 1344:300-311

Senthilkumar R, Yeh KW (2012) Multiple biological functions of sporamin related to stress tolerance in sweet potato (Ipomoea batatas Lam). Biotechnol Advances 30:1309-1317

Shewry PR (2003) Tuber storage proteins. Ann Bot 91:755-769

Sohonie K, Bhandarker AP (1954) Trypsin inhibitors in Indian foodstuffs: I. Inhibitors in vegetables. J Sci Ind Res 13B:500-503

Trümper S, Follmann H, Häberlein I (1994) A novel dehydroascorbate reductase from spinach chloroplasts homologous to plant trypsin inhibitor. FEBS Lett 352:159-162

Wu CH, Lin JA, Hsieh WC, Yen GC (2009) Low-density-lipoprotein (LDL)-bound flavonoids increase the resistance of LDL to oxidation and glycation under pathophysiological concentrations of glucose in vitro. J Agric Food Chem 57:5058-5064

Yeh KW, Chen JC, Lin MI, Chen YM, Lin CY (1997) Functional activity of sporamin from sweet potato (Ipomoea batatas Lam.): a tuber storage protein with trypsin inhibitory activity. Plant Mol Biol 33:565-570

\section{Publisher's Note}

Springer Nature remains neutral with regard to jurisdictional claims in published maps and institutional affiliations.

\section{Submit your manuscript to a SpringerOpen ${ }^{\circ}$ journal and benefit from:}

- Convenient online submission

- Rigorous peer review

- Open access: articles freely available online

- High visibility within the field

- Retaining the copyright to your article

Submit your next manuscript at $\boldsymbol{\nabla}$ springeropen.com 